Regular Article

\title{
Serrated flow in nanostructured binary Mg-Al alloys
}

\author{
M. Pozuelo*, Y.W. Chang, J. Marian, J.M. Yang \\ Department of Materials Science and Engineering, University of California Los Angeles, Los Angeles, CA 90095, USA
}

\section{A R T I C L E I N F O}

Article history:

Received 2 August 2016

Received in revised form 14 September 2016

Accepted 15 September 2016

Available online $\mathrm{xxxx}$

Keywords:

Mechanical milling

In-situ SEM micro-compression tests

Magnesium alloys

Portevin-Le Chetelier effect

\begin{abstract}
A B S T R A C T
Serrated flow, also known as the Portevin-Le Chatelier effect, has been extensively investigated in binary Mg-alloys such as Mg-Ag, Mg-Y and Mg-Li alloys. Interestingly, however, this effect has not been observed in Mg-Al alloys. In this work, we report the appearance of serrations in nanostructured Mg-Al alloys processed by cryomilling and spark-plasma-sintering during in-situ SEM microcompression tests at room temperature and strain rates between $2 \times 10^{-3}$ and $10^{-1} \mathrm{~s}^{-1}$. The observed serrated stress-strain behavior is attributed to the dynamic strain aging by which interstitial $\mathrm{O}$ and/or $\mathrm{N}$ impurities introduced during cryomilling diffuse and interact with in-grown dislocations during deformation.
\end{abstract}

(c) 2016 Acta Materialia Inc. Published by Elsevier Ltd. All rights reserved.
The Portevin-Le Chatelier (PLC) effect is one of the best studied plastic instabilities in metallic materials. One of its main manifestations, in the appropriate strain rate and temperature range, is the appearance of "serrated flow" in the stress-strain curve. The PLC effect has been investigated extensively, from both experimental and theoretical points of view, in body and face centered cubic materials. However, the study of this phenomenon in hexagonal close-packed (hcp) materials, particularly in binary Mg-Al alloys, has been limited.

Chaturvedi et al. [1] reported serrated flow in a binary $\mathrm{Mg}-\mathrm{Ag}$ alloy in the $53-124^{\circ} \mathrm{C}$ temperature range, and attributed the locking of mobile dislocations to the diffusion of silver atoms during deformation. Serrated flow was also observed by Gao et al. [2] in binary Mg-Y alloys during tensile deformation between 150 and $250{ }^{\circ} \mathrm{C}$. In contrast, Caceres et al. [3] investigated the serrated behavior of as-cast binary Mg-Al alloys and did not observe serrations. Interestingly, Standford et al. [4] reported flow serrations during deformation of a Mg-Gd alloy at $200^{\circ} \mathrm{C}$. However, they could not observe serrations in a $\mathrm{Mg}$-Al alloy processed under the same conditions to obtain a similar extrusion texture and grain size.

The PLC effect has also been found in ternary Mg-alloys, such as AZ91 by Corby et al. [5], and in alloys containing rare earth additions such as Mg-Y-Nd by Zhu et al. [6], Mg-Gd-Zn alloys by Wu et al. [7] and Mg-Mn-Nd by Dudamell et al. [8]. In all these systems, serrated flow was attributed to the dynamic interaction between solute atoms and dislocations, a phenomenon known as dynamic strain aging (DSA), first proposed by Cottrell in 1953 [9]. In the case of AZ91 at room temperature, Corby et al. [5] reported that both $\mathrm{Al}$ and $\mathrm{Zn}$ atoms were involved in the DSA. They suggested that Al might be the

\footnotetext{
* Corresponding author.

E-mail addresses: pozuelo@ucla.edu, marta.pozuelo@gmail.com (M. Pozuelo).
}

dominant solute, while $\mathrm{Zn}$ acted as a catalyzer enabling the formation of a forest of dislocations through which $\mathrm{Al}$ atoms might pipe diffuse.

This study reveals the appearance of serrations at room temperature in a nanostructured binary $\mathrm{Mg}-10 \mathrm{Al}$ (wt.\%) alloy processed via cryomilling and spark plasma sintering (SPS). Our previous work has demonstrated that cryomilling (mechanical milling in liquid nitrogen atmosphere) is an optimum severe plastic deformation technique to obtain nanocrystalline-sized grains in binary Mg-Al alloys [10,11]. As a consolidation technique, the SPS method allows cryomilled Mg-powders to be synthesized in a very short time into a bulk nanostructured material with a characteristic bimodal grain distribution [10-12]. This feature results in a simultaneous enhancement of the strength and ductility [13-15]. Coarse grains facilitate dislocation mobility and contribute to the ductility, while nano-sized grains contribute to the strengthening by the Hall-Petch mechanism. Additionally, we have demonstrated that small amounts of impurity elements may be introduced during cryomilling in liquid nitrogen and form nanoscale dispersoids such as oxides and nitrides. Indeed, thorough TEM analyses of the cryomilled powders have revealed some traces of nitrogen and oxygen atoms [10]. The presence of second phases, however, is known to play a significant role in stabilizing the microstructure after heat treatment [16].

Although to the best of our knowledge serrations have not been reported for binary Mg-Al alloys [3,4], if serrations are indeed associated with a solute atom-dislocation interactions, they should logically also occur in our nanostructured Mg-10Al alloy, as all the ingredients for the PLC effect to occur are also present by design. The idea that impurity atoms introduced during cryomilling might promote DSA during mechanical testing was also hypothesized for a nanostructured Al alloy processed by cryomilling [17]. This work is motivated by this hypothesis, and is focused on in-situ nanomechanical testing to understand the 
underlying mechanisms leading to serrated flow in binary nanostructured $\mathrm{Mg}$-Al alloys.

Pure $\mathrm{Mg}$ and $\mathrm{Al}$ powders were blended to formulate the final composition Mg-10Al (wt.\%) alloy. Cryomilling was performed for $8 \mathrm{~h}$ in liquid nitrogen atmosphere. Spark-plasma-sintering (SPS) process was carried out in vacuum for $5 \mathrm{~min}$ at $400{ }^{\circ} \mathrm{C}$ and under uniaxial pressure of $100 \mathrm{MPa}$ to consolidate the cryomilled powders. More details about the material processing can be found in refs. [10,11].

In order to evaluate the mechanical behavior of nanostructured $\mathrm{Mg}$ 10Al alloys under different strain rate conditions, in-situ SEM microcompression experiments were conducted at room temperature under displacement control mode and at a strain rates between $5 \times$ $10^{-4}$ and $10^{-1} \mathrm{~s}^{-1}$. A PI 85 SEM PicoIndenter (Hysitron Inc.) with a 5 - $\mu \mathrm{m}$ flat punch diamond probe inside a FEI Nova 600 Nanolab DualBeam Focused Ion Beam-Scanning Electron Microscope (FIB-SEM) was used for these experiments. Micropillars of $4-\mu \mathrm{m}$ in diameter and an aspect ratio of 1:3 were machined from the sintered samples by FIB operated at $30 \mathrm{kV}$. A series of concentric annular milling patterns with different currents were applied. In order to tailor the pillars into the desired shape and minimize the tapering $[18,19]$, a low beam current $(0.3 \mathrm{nA})$ was used as final milling step.

Fig. 1 shows the results from in-situ SEM microcompression tests at room temperature of a nanostructured binary $\mathrm{Mg}-10 \mathrm{Al}$ alloy. The plot in Fig. 1a shows representative engineering stress-strain curves at different strain rates for $\mathrm{Mg}-10 \mathrm{Al}$ micropillars of $4 \mu \mathrm{m}$ in diameter. A minimum of three tests per strain rate were performed but only one curve is shown here for clarity. We find that serrations start to appear at a strain rate of $2 \times 10^{-3} \mathrm{~s}^{-1}$ with a remarkable serrated effect occurring at $2 \times 10^{-2} \mathrm{~s}^{-1}$ (red curve). These results can be better seen in a schematic representation of strain rate as a function of the inverse of temperature (Fig. 1b), where the two straight lines mark the boundaries of the region where serrations occur. All strain rates shown in Fig. 1a have been highlighted with the same color code at room temperature in this figure. Serrations are classified as of type A, B, or C, depending on applied load, strain rate, and temperature $[20,21]$. Type B serrations that are characterized by regular oscillations around the general level of the stress-strain curve are observed at all strain rates. A transition from type B to type $C$, which is characterized by abrupt load drops below the general level of the stress-strain curve can be also observed at $10^{-1} \mathrm{~s}^{-1}$ of strain rate. (See Supplementary Fig. 1 for a better observation of the serrations).

Fig. 1c displays the average yield stress as a function of strain rate with the standard deviation of the average as error bars. It is worth noting the abnormal strain rate sensitivity (SRS) at room temperature, which is positive for lower strain rates and negative for strain rates higher than $2 \times 10^{-2} \mathrm{~s}^{-1}$. This has also been reported for a ternary $\mathrm{Mg}$-Li-Al alloy [22] and rationalized in terms of the competition between two mechanisms: shearing of precipitates by dislocations (leading to a positive SRS) at lower strain rates [23,24]; and DSA (resulting in a negative SRS) at higher strain rates [25].

The negative SRS at room temperature caused by DSA is considered as a sufficient condition for serrated flow in different material systems $[5,9,22,25-27]$. The other mechanism of shearing of precipitates by dislocations that was firstly reported by Zambo et al. [28], Kumar et al. [29], Tian et al. [23], Wu et al. [24], and recently by Choudhuri et al. [30] tends to be suitable for alloys with a large number of fine precipitates in the matrix. As the deformation progresses, substantial pileups of dislocations may shear the fine precipitates, and then the stress concentration can be relaxed temporarily. Thus, repeated shearing can lead to successive stress drops resulting in stress-strain curves with a jerky appearance. From our previous work [11], we have evidence that $\gamma-\mathrm{Al}_{12} \mathrm{Mg}_{17}$ precipitates are primarily obstacles for dislocation movement contributing to the enhancement of strength in a Mg-10Al alloy. Therefore, the presumption that these precipitates act as shearable objects contributing to the PLC effect by repeated shearing [9,23-24,28-30] is not substantiated by strength measurements.

Additionally, it is interesting to point out a possible relationship between the sign of the SRS slope and the grain size. A high SRS has been reported to be related to the nanocrystalline grain size [31,32]. For instance, Schwaiger et al. [31] found that nanocrystalline pure Ni exhibited a positive SRS in flow stress, an effect that was not found in ultrafine and microcrystalline Ni. Fan et al. [33] and Joshi et al. [34] observed the PLC effect in ultrafine-grained Al-alloys only over a small strain range immediately following yield, while the coarse-grained alloy exhibited serrated flow over nearly the entire plastic strain range for the same applied displacement rate. Del Valle and Ruano [35] reported that SRS strongly increases by decreasing grain size below $15 \mu \mathrm{m}$ in a $\mathrm{Mg}-\mathrm{Al}-$ $\mathrm{Zn}$ alloy at moderate temperatures.

In view of the bimodal grain size distribution displayed by our specimens after SPS [11], we speculate that serrated flow is governed by the competition between positive SRS contributed by nanocrystalline grains $(\sim 35 \mathrm{~nm})$ at low strain rates (caused by GB strengthening mechanisms) and negative SRS originating in coarse grains $(\sim 400 \mathrm{~nm})$ from DSA. Although this explanation is somewhat speculative, our argument is that true DSA can only fully develop in coarse grained systems. In very fine grained materials, dislocation/grain boundary interactions and other grain boundary effects are likely to govern plastic flow, not leaving enough room for solute diffusion and/or dislocation glide to develop to the extent required by DSA.

Fig. 2 shows as an example two characteristic nanostructured Mg$10 \mathrm{Al}$ micropillars before and after microcompression at different strain rates. Left images in Fig. 2a and b show $52^{\circ}$ tilted-view SEM images of those micropillars before microcompression. All micropillars are slightly tapered as a result of the annular cutting method by FIB. However, we
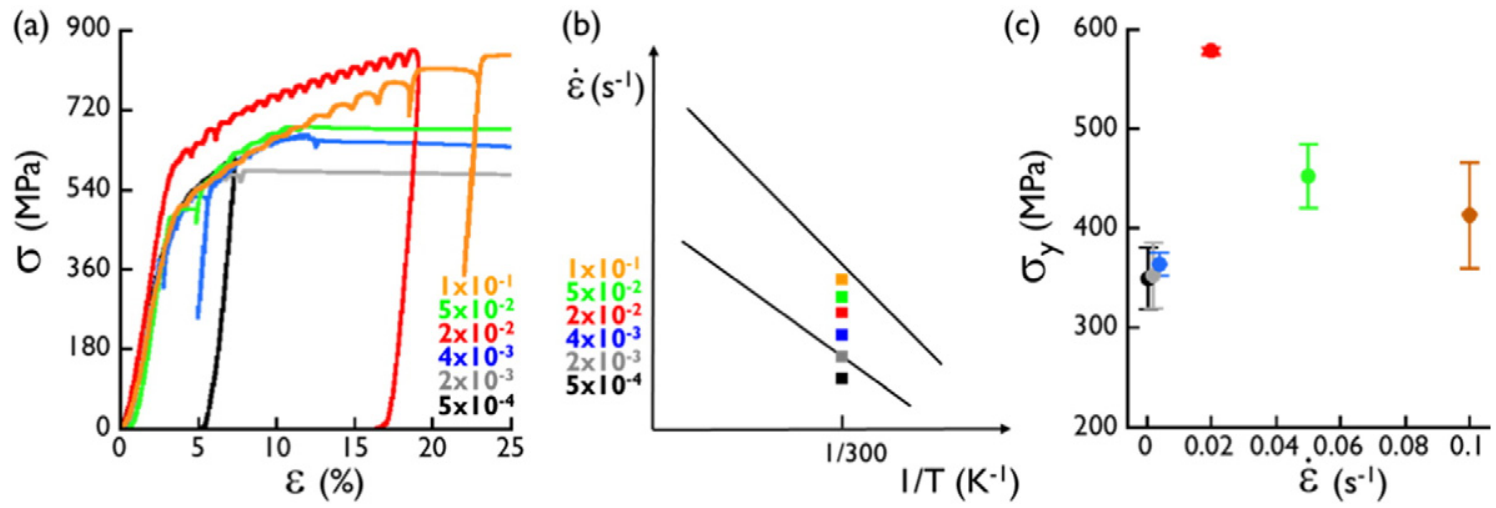

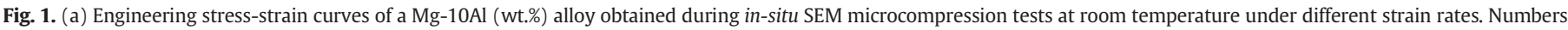

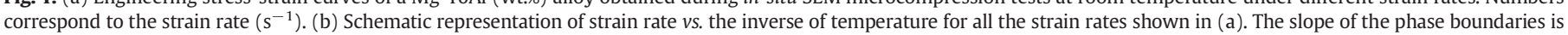
just schematic and may not necessarily coincide with the true trend. (c) Average yield stress as a function of strain rate from the data in (a). 
(a)

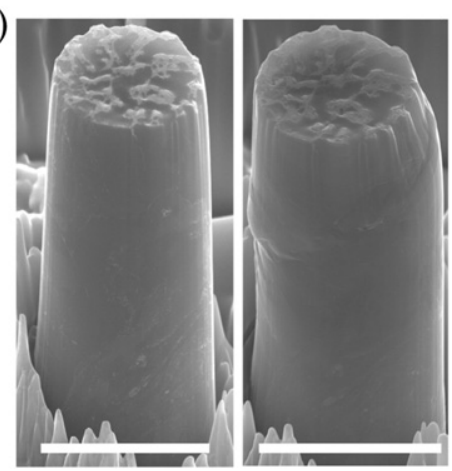

(b)

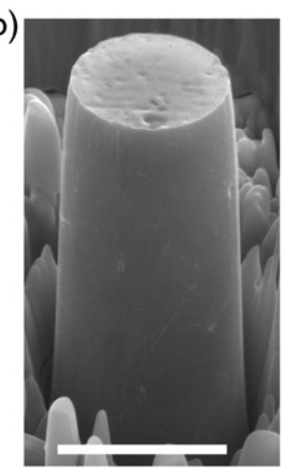

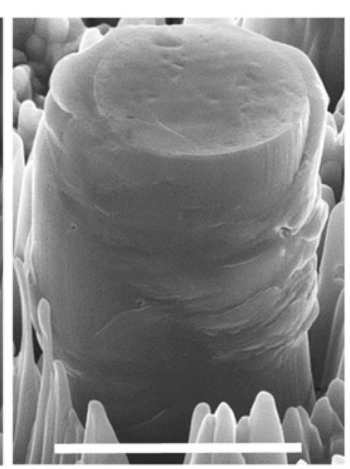

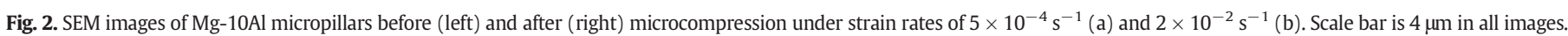

achieved angles $\leq 1.5^{\circ}$, which are smaller than the values reported in the literature $\left(2-5^{\circ}\right)$ for typical micropillars machined by FIB [36]. In any case, to avoid any overestimation of the measured stress due to the tapering, the diameter is considered at half-height of the micropillar ( $4 \mu \mathrm{m})$. Micropillar size was also carefully selected $\sim 4 \mu \mathrm{m}$ to avoid sizeinduced strengthening, as we have demonstrated in previous works [36,37], and to provide results comparable with those from macro scale tests of Mg alloys. The images on the right hand side of Fig. 2a and b show $52^{\circ}$ tilted-view SEM images of the compressed micropillars. Deformation bands are apparent on the micropillars' surface, especially after increasing the strain rate up to $2 \times 10^{-2} \mathrm{~s}^{-1}$. This observation suggests a direct correlation between the DSA and the macroscopic response of the micropillar.

A detailed analysis of the serrations at $2 \times 10^{-2} \mathrm{~s}^{-1}$ of strain rate is shown in the Supplementary Video 1 and Fig. 3. From the video, we can correlate SEM images of the micropillar under compression with its loading-displacement curve. In addition to the deformation bands on the micropillar surface corresponding to the serrated flow in the loading-displacement curve, a thickening of the micropillar diameter is observed during compressive loading. Specifically, an increase of $\sim 18 \%$ is measured for the diameter at half-height of the micropillar after the compression test is completed.

Fig. 3a shows the engineering stress-strain curve (red) along with the true stress-strain data by interpolation (grey curve). As expected from the increase of pillar diameter during microcompression, the apparent work-hardening effect from the engineering stress-strain curve is no longer visible in the true stress-strain curve. Typical type B serrations are shown in Fig. 3a. Our results suggest that this type of serrations appear when a critical stress is applied and begins with a pinning process. That means that dislocations accumulate some degree of slip prior to interacting with solute atoms, which then results in the first stress drop that gives rise to a serration. From the stress-strain curve, the critical stress and strain to induce serrated flow are around $600 \mathrm{MPa}$ and 3.6\%, respectively. This type B serration can be better seen in Fig. $3 \mathrm{~b}$, where the two main processes, i.e. pinning and unpinning of dislocations by solute atoms are clearly identified. We assume that at this particular strain rate, the diffusivity of solute atoms might become similar to the dislocation mobility, leading first to dislocation pinning and stress buildup, which is in turn followed by dislocation unpinning and rapid stress drop. The stress drop, $\Delta \sigma$, and strain increment, $\Delta \varepsilon$, are represented as a function of the number of drops in Fig. 4. Interestingly, $\Delta \sigma$ follows a linear relationship with the number of drops (Fig. 4a) while $\Delta \varepsilon$ is nearly constant at approximately $0.9 \%$ (Fig. 4b). A linear relationship is also found between $\Delta \sigma / \sigma_{\min }$ and the strain $\varepsilon$ (Fig. 4c), which indicates that the linearity between $\Delta \sigma$ and the number of drops is not simply an artifact of the engineering stress-strain curve. Since the stress drop can be related to the size of plastic events [20], the linear relationship of $\Delta \sigma$ with the number of drops suggests that the shear displacement of the deformation bands linearly increases with the number of serrations.

Next we provide some micromechanical analysis of our results. DSA is brought about by dislocation solute-interactions, the nature of which depends critically on the type of solute (and dislocation). To begin our analysis, we hypothesize that the solutes present in our system in addition to substitutional $\mathrm{Al}$ atoms are interstitial $\mathrm{O}$ and $\mathrm{N}$ atoms introduced during cryomilling. To test this hypothesis and ascertain which of these atomic species -substitutional solutes or interstitial impurities- is responsible for DSA, we calculate the characteristic lengths of diffusion and slip, respectively, of atoms and dislocations in our deformation tests. Unfortunately, there are no reliable data for diffusion of oxygen
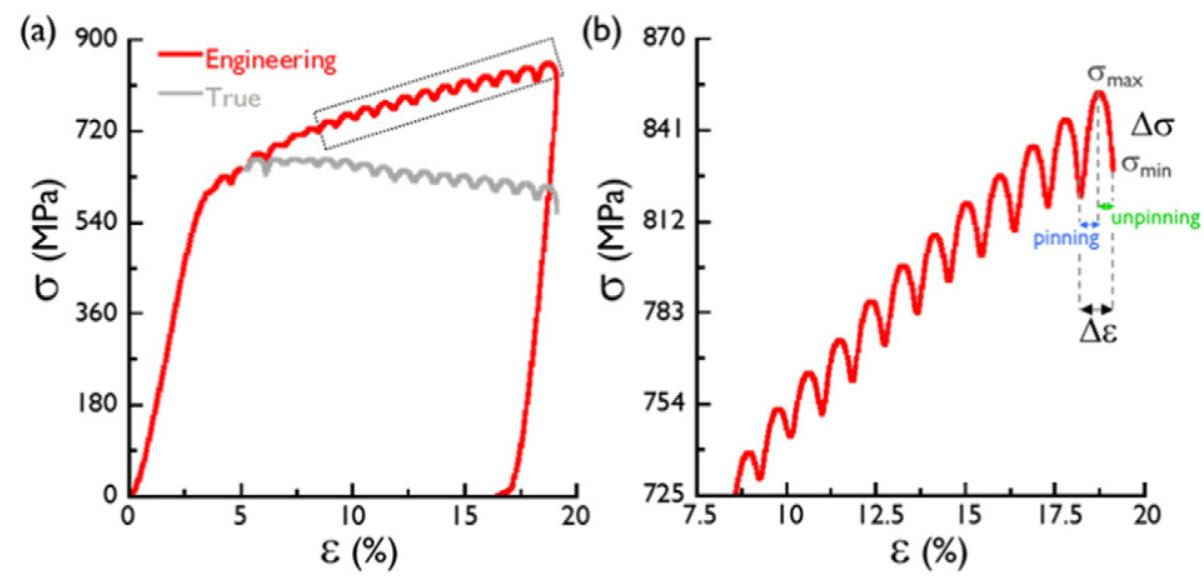

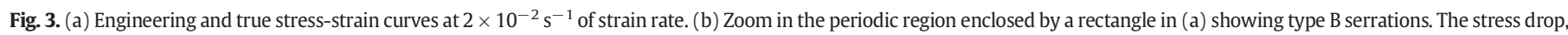
$\Delta \sigma$, and the strain increment, $\Delta \varepsilon$, along with the pinning and unpinning regions are highlighted in this plot. 

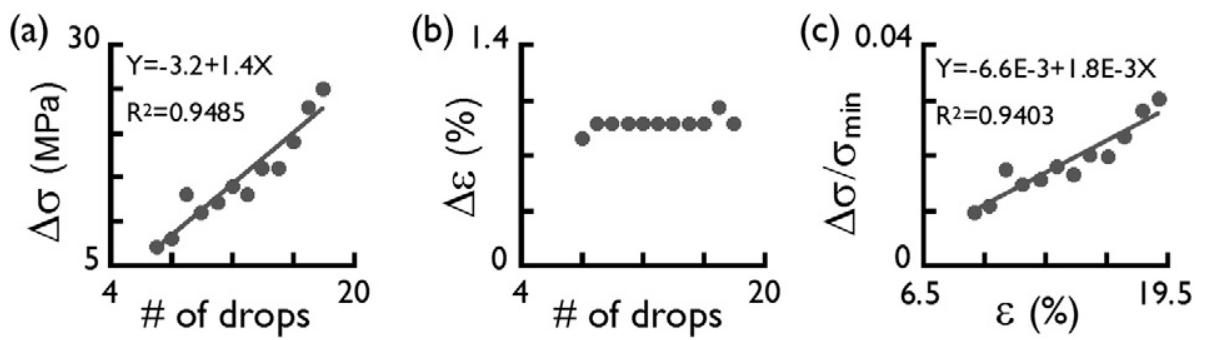

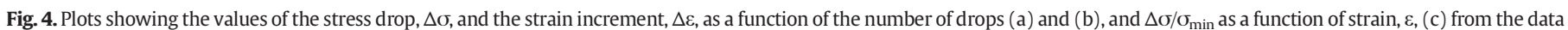
shown in Fig.3.

and/or nitrogen in $\mathrm{Mg}$ or Mg-Al alloys in the literature. Thus, we carry out the analysis for Al and test whether it can be eliminated as the solute responsible for DSA.

The characteristic slip distance travelled by a dislocation during one of the teeth of the stress-strain curve serrations can be obtained from Orowan's equation [38]:

$\dot{\varepsilon}=b \rho v$

where $\dot{\varepsilon}$ is the strain rate, $b$ is the Burgers vector and $v$ is the dislocation velocity. From Eq. (1):

$l_{d}=\frac{\dot{\varepsilon} \delta t}{b \rho}=\frac{\Delta \varepsilon}{b \rho}$

with $\delta t$ being the time duration of one of the serrations, estimated as $\delta t$ $=\Delta \varepsilon / \dot{\varepsilon}$. For a strain rate of $2 \times 10^{-2} \mathrm{~s}^{-1}$ and with $\Delta \varepsilon=9 \times 10^{-3}$ from Fig. 4 , we have $\delta t=0.45 \mathrm{~s}$. Next, with a value of $b=3.21 \times 10^{-10} \mathrm{~m}$ for basal dislocations and an upper bound for the dislocation density of $\rho$ $10^{16} \mathrm{~m}^{-2}$, we obtain $l_{d} \approx 3 \times 10^{-9} \mathrm{~m}$.

For its part, the characteristic diffusion length of Al solute atoms diffusing in $\mathrm{Mg}, l_{s}$, can be calculated as:

$l_{s} \approx \sqrt{D \delta t}=\sqrt{D_{o} \exp \left(\frac{-E_{a}}{R T}\right) \delta t}$

where $D$ is the diffusion coefficient, $D_{o}$ is the diffusivity pre-factor, $R$ is Boltzmann's constant, $T$ is the absolute temperature, and $E_{a}$ is the activation energy for diffusion. Using values reported in the literature of $D_{o}=$ $3.9 \times 10^{-3} \mathrm{~m}^{2} / \mathrm{s}$ and $E_{a}=155 \mathrm{~kJ} / \mathrm{mol}$ [39], we get $l_{s} \approx 10^{-15} \mathrm{~m}$ at room temperature and under the same strain rate condition.

Given the six order of magnitude discrepancy found between the estimated values of $l_{d}$ and $l_{s}$ for $\mathrm{Al}$ atoms, we conclude that the diffusion of $\mathrm{Al}$ atoms in $\mathrm{Mg}$ at a strain rate of $\dot{\varepsilon}=2 \times 10^{-2} \mathrm{~s}^{-1}$ and room temperature cannot explain the observed PLC effect in our experiments. Therefore, by elimination, we attribute the DSA mechanism in nanostructured binary $\mathrm{Mg}$-Al alloys to interstitial $\mathrm{O}$ and/or $\mathrm{N}$ impurities which are likely to diffuse at much higher rates than $\mathrm{Al}$ solute atoms and thus result in a dynamic interaction with dislocations.

In summary, we report the PLC effect observed for the first time in binary Mg-Al alloys during in-situ SEM microcompression tests at room temperature between $2 \times 10^{-3}$ and $10^{-1} \mathrm{~s}^{-1}$ of strain rate. At $2.0 \times 10^{-2} \mathrm{~s}^{-1}$ of strain rate, type B serrations occur after reaching a critical stress of around $600 \mathrm{MPa}$. Additionally, the stress drop follows a linear relationship with the number of drops suggesting that the shear displacement of the deformation bands linearly increases with the number of serrations. We attribute the abnormal SRS (positive for lower strain rates and negative for strain rates higher than $2 \times 10^{-2}$ $\mathrm{s}^{-1}$ ) to the bimodal grain size distribution characteristic of nanostructured $\mathrm{Mg}$-Al alloys processed by SPS. Finally, we rationalize the PLC effect in nanostructured Mg-Al alloys in terms of the diffusivity of interstitial $\mathrm{O}$ and/or $\mathrm{N}$ impurities (introduced during cryomilling) and their interaction with dislocations.

Supplementary data to this article can be found online at http://dx. doi.org/10.1016/j.scriptamat.2016.09.019.

\section{Acknowledgements}

This work was funded by the Army Research Office (ARO) under contract No. W911NF-09-1-0558. The authors are particularly grateful to ARO program manager, Dr. Larry Russell for his supervision and to Christopher Melnyk (California Nanotechnologies Inc.) for his technical assistance.

\section{References}

[1] M. Chaturvedi, D.J. Lloyd, K. Tangri, Met. Sci. 6 (1972) 16.

[2] L. Gao, R.S. Chen, E.H. Han, J. Alloys Comp. 472 (2009) 234

[3] C.H. Cáceres, D.M. Rovera, J. Light Met.s 1 (2001) 151.

[4] N. Standford, I. Sabirov, G. Sha, A. La Fontaine, S.P. Ringer, M.R. Barnett, Metal. Mater. Trans. 41A (2010) 734

[5] C. Corby, C.H. Cáceres, P. Luká, Mater. Sci. Eng. A387 (2004) 22.

[6] S.M. Zhu, J.F. Nie, Scr. Mater. 50 (2004) 51.

[7] D. Wu, R.S. Chen, E.H. Han, Mater. Sci. Eng. A 532 (2012) 267.

[8] N.V. Dudamell, P. Hidalgo-Manrique, A. Chakkedath, Z. Chen, C.J. Boehlert, F. Gálvez, S. Yi, J. Bohlen, D. Letzig, M.T. Pérez-Prado, Mater. Sci. Eng. A583 (2013) 220.

[9] A.H. Cottrell, Philos. Mag. 44 (1953) 829.

[10] M. Pozuelo, C. Melnyk, W.H. Kao, J.-M. Yang, J. Mater. Res. 26 (2011) 904

[11] M. Pozuelo, Y.W. Chang, J.-M. Yang, Mater. Sci. Eng. A 594 (2014) 203.

[12] M.J. Zehetbauer, Y.T. Zhu, Bulk Nanostructured Materials, Wiley-VCH, Weinheim, 2009

[13] B.Q. Han, Z. Lee, D. Witkin, S. Nutt, E.J. Lavernia, Metall. Mater. Trans. 36 A (2005) 957.

[14] Y. Wang, M. Chen, F. Zhou, E. Ma, Nature 419 (2002) 912.

[15] S.R. Bakshi, D. Lahiri, A. Agarwal, Int. Mater. Rev. 55 (2010) 41.

[16] V.L. Tellkamp, A. Melmed, E.J. Lavernia, Metall. Mater. Trans. A 32 (2001) 2335

[17] B.Q. Han, J.Y. Huang, Y.T. Zhu, E.J. Lavernia, Scr. Mater. 54 (2006) 1175.

[18] H. Zhang, B.E. Schuster, Q. Wei, K.T. Ramesh, Scr. Mater. 54 (2006) 181

[19] S. Shim, H. Bei, M.K. Miller, G.M. Pharr, E.P. George, Acta Mater. 57 (2009) 503.

[20] K. Chihab, Y. Estrin, L.P. Kubin, J. Vergnol, Scr. Metal. 21 (1987) 203.

[21] W.H. Wang, D. Wu, S.S.A. Shah, R.S. Chen, C.S. Lou, Mater. Sci. Eng. A649 (2016) 214.

[22] C. Wang, Y. Xu, E. Han, Mater. Lett. 60 (2006) 2941

[23] B.H. Tian, Y.G. Zhang, C.Q. Chen, Mater. Sci. Eng. A 254 (1998) 227.

[24] D. Wu, R.S. Chen, E.H. Han, Mater. Sci. Eng. A 532 (2012) 267.

[25] S.K. Wu, C. Chien, C.S. Yang, H.Y. Bor, Mater. Sci. Eng. A605 (2014) 33.

[26] R.C. Picu, Acta Mater. 52 (2004) 3447.

[27] L. Jianga, J.J. Jonas, R. Mishra, Mater. Sci. Eng. A528 (2011) 6596.

[28] S.J. Zambo, J.A. Wert, Scripta Metall. Mater. 29 (1993) 1523.

[29] S. Kumar, E. Pink, Scripta Metall. Mater. 32 (1995) 749.

[30] D. Choudhuri, S.A. Mantri, T. Alama, S. Banerjee, R. Banerjee, Scr. Mater. 124 (2016) 15.

[31] R. Schwaiger, B. Moser, M. Dao, N. Chollacoop, S. Suresh, Acta Mater. 51 (2003) 5159

[32] Q. Wei, S. Cheng, K.T. Ramesh, E. Ma, Mater. Sci. Eng. A 381 (2004) 71.

[33] G.J. Fan, G.Y. Wang, H. Choo, P.K. Liaw, Y.S. Park, B.Q. Han, E.J. Lavernia, Scr. Mater. 52 (2005) 929.

[34] S.P. Joshi, C. Eberl, B. Cao, K.T. Ramesh, K.J. Hemker, Exp. Mech. 49 (2009) 207.

[35] J.A. del Valle, O.A. Ruano, Scr. Mater. 55 (2006) 775.

[36] Y.W. Chang, M. Pozuelo, J.-M. Yang, Mater. Res. Lett. 2 (2014) 199.

[37] M. Pozuelo, Y.W. Chang, J.M. Yang, Mater. Sci. Eng. A 633 (2015) 200.

[38] E. Orowan, Discussion in Symposium on Internal Stresses in Metals and Alloys, Institute of Metals, London, 1948451.

[39] S. Brennan, A.P. Warren, K.R. Coffey, N. Kulkarni, P. Todd, M. Kilmov, Y. Sohn, JPED 33 (2012) 121 